\title{
Multimodality Imaging of Right-Sided (Tricuspid Valve) Papillary Fibroelastoma: Recognition of a Surgically Remediable Disease
}

\author{
Shantanu V. Srivatsa Prabhat Adhikari Pervaiz Chaudhry \\ Sanjay S. Srivatsa \\ Department of Cardiology and Cardiothoracic Surgery, Community Regional Medical \\ Center, Fresno, Calif., USA
}

\section{Key Words}

Cardiac tumors $\cdot$ Papillary fibroelastoma $\cdot$ Imaging

\begin{abstract}
Presentation of an increasingly recognized right-sided primary valve tumor of clinical importance: the tricuspid valve papillary fibroelastoma (PF). Early recognition and surgical intervention is emphasized for valvular PF, which carries a significant risk of morbidity and mortality. Newer imaging techniques, including CT and MRI, assist in localizing and differentiating PF from alternative cardiac pathology.

(C) 2013 S. Karger AG, Basel
\end{abstract}

\section{Case Presentation}

A 61-year-old Hispanic woman presented with recurrent paroxysmal pleuritic chest pain and severe dyspnea. Between episodes, the patient was normally functional and asymptomatic. There was no prior history of structural cardiac, coronary, pulmonary, or systemic disease. Pulmonary CT angiography did not reveal any emboli. Transthoracic and transesophageal echocardiography showed a mobile mass of moderate size and shimmering edges, measuring $1.3 \times 0.9 \times 1.1 \mathrm{~cm}$, attached to the septal tricuspid valve (TV) leaflet. Pulmonary pressure was minimally elevated at 35-40 $\mathrm{mm} \mathrm{Hg}$. Anticoagulation with rivaroxaban (oral factor Xa inhibitor) was initiated. A pedunculated mass with peripheral shimmering frond-like appearance and stalk attached to the septal TV leaflet was seen prolapsing through the TV with tricuspid regurgitation (fig. 1A, B). CT pulmonary angi-

Sanjay S. Srivatsa, MD, FACC

Department of Cardiology and Cardiothoracic Surgery

Community Regional Medical Center

Fresno, CA 93721 (USA)

E-Mail sanjaysrivatsa@hotmail.com 
ography did not show gross filling defects to suggest pulmonary embolism (PE) but revealed a mobile mass visible in the orthogonal image planes (fig. 2A, B). Cardiac MRI revealed a mobile mass measuring $\geq 1 \mathrm{~cm}$ in multiple dimensions, showing hyperintensity on T2weighted and inversion recovery sequences (fig. 3A-D). A differential diagnosis of papillary fibroelastoma (PF) versus cardiac myxoma was entertained. Cardiac surgery was advised due to size $(>1 \mathrm{~cm})$, symptomatology, and the established recurrent embolic potential of the tumor. At surgery, shave excision of the sessile mass, and TV repair using a 30-mm Medtronic Contour ring was performed. Gross pathology revealed a friable mass with surface fronds on a short pedicle, with the typical sea anemone appearance of a PF. Microscopic findings showed avascular branching filamentous fronds with a single endothelial cell covering. Each frond had a fibrous core with loose connective tissue rich in mucopolysaccharide (fig. 1C, D). Postoperatively, the patient continued anticoagulation for 8 weeks. To date, the patient remains asymptomatic at rest and exercise.

\section{Discussion}

PF accounts for $\sim 10 \%$ of all primary cardiac tumors. Of these, right-sided valvular (tricuspid and pulmonary) PF are relatively rare presentations of an increasingly recognized benign (non-metastatic) though clinically important entity. Histopathologically, it is considered a hamartomatous entity with endothelial genesis secondary to turbulent highstress flow and secondary endothelial dysfunction with proliferation [1]. Iatrogenic cardiac trauma and virus-induced tumor genesis have been proposed. The majority $(75 \%)$ of fibroelastomas arise from the valvular endothelium, and are left-sided in origin [1]. Rightsided PFs are an important potentially fatal cause of sudden cardiac death, syncope, chest pain, and dyspnea. While PFs are slow growing, their propensity to accumulate superficial thrombus and fragment, embolizing tumor or thrombus fragments into the distal pulmonary circulation or paradoxically embolizing to the left-sided circulation, is significant $[2,3]$. Clinical recognition of these tumors is therefore important [4-6]. While echocardiographic imaging remains the most prevalent gold standard in use clinically, CT chest and cardiac magnetic resonance (CMR) are both increasingly used. Imaging right-sided PFs is important due to the delayed presentation of right-sided versus left-sided tumors. While highresolution or thin-section multislice CT of the chest offers excellent spatial and temporal resolution, the issues of contrast and radiation exposure remain. We emphasize the incremental value of CMR in PF recognition because it affords versatility of multiplane reconstruction and pathological discrimination based on soft-tissue contrast resolution of water and fat content using T1-/T2-weighted imaging [7]. The differential diagnosis of PF includes myxomas, thrombus, or infectious endocarditis vegetations [1]. Steady-state free precession sequences revealed a hyperintense highly mobile pedunculated mass attached to the septal TV (fig. 3A). PF CMR features include isointense T1-imaging and hyperintense T2imaging. In contrast, the thrombus is hypointense on T1- or T2-imaging, while myxomas have isointense T1- and high T2-signal intensity due to their high extracellular water content. FIESTA (bright blood imaging) MRI sequences of PF show a sharply delineated pedunculated mobile mass with: (1) hyperintense signal relative to the myocardium but hypointensity relative to the blood pool (fig. 3A) and (2) hyperintense signal by inversion recovery T2 sequences and delayed gadolinium hyperenhancement (fig. 3C, D). Even the non-high resolution CT pulmonary angiogram clearly demonstrated the TV-PF (fig. 2). FIESTA (fast imaging employing steady-state acquisition) is an ultrafast MRI technique based on balanced steady-state free precession that offers high-signal-to-noise ratio and 
Srivatsa et al:: Multimodality Imaging of Right-Sided (Tricuspid Valve) Papillary Fibroelastoma: Recognition of a Surgically Remediable Disease

excellent image contrast. Inversion recovery sequences, offer differentiation of tumor from fat or muscle by the additive signal intensity effects of prolonged T1 and T2, and nulling fat signal (fig. 3C). Early gadolinium enhancement (post-contrast T1-weighted images, fig. 3D) is seen in $40-50 \%$ of benign tumors and varies with capillary permeability or tumor vascularity [7].

\section{Conclusion}

Left-sided PFs embolize to the coronary, cerebral or systemic circulation. Less well appreciated is the morbidity of right-sided PFs. Their slow growth pattern and more insidious and life-threatening presentations are noteworthy. Surgical excision appears safe, potentially curative (however, good long-term follow-up data for recurrence is lacking), and is advised where symptoms or large PF tumor size or significant tumor mobility is present [4-7]. There are documented cases of TV-PF with PE [2,3]. Pleuritic chest pain and dyspnea symptoms are common, and may reflect recurrent distal pulmonary circulation embolization, despite overtly negative CT and V/Q scan findings for proximal PE. In the largest surgical series of 88 patients (93 PF lesions) right-sided PFs accounted for $10 \%$ of all valvular (TV, PV) lesions and 16\% of all cardiac chamber (RA, RV) lesions [4]. Mean age at presentation was $60 \pm 14$ years, $83 \%$ required only a shave excision, with $9 \%$ requiring shave excision and valve repair. Interestingly, right-sided tumor burden was greater than left-sided, presumably reflecting the more indolent growth and presentation of these lesions. Reviewing 29 lesions (23 patients), Anastacio et al. [5] suggested an 18\% incidence of rightsided valvular lesions. PE from a right atrial and pulmonary valve PF was documented. Later, this patient developed a new left-sided aortic valve PF 3 months after successful right-sided tumor removal. In a meta-analysis of 725 cases of cardiac PF (CPF) in 2003, Gowda et al. [6] identified tumor mobility as the independent predictor of PF-related death or non-fatal embolization. They suggested an $11 \%$ rate of right-sided valvular PFs, emphasizing the possibility of multiple tumor locations within a single patient. Most CPF-related death occurred from obstruction of the aortic valve or coronary embolization of left-sided tumors. However, one death occurred from RV outflow obstruction, and two non-fatal embolizations of the pulmonary circulation resulted. Gowda et al. [6] identified 25 patients with CPF managed medically with anticoagulation alone. Of these, 12 died of PF-related causes including coronary embolization or obstruction (11 cases), 4 developed non-fatal distal embolization (2 coronary, 1 cerebral 1 pulmonary) despite anticoagulation. For these reasons, many advocate early surgical intervention for symptomatic (obstruction /valvular dysfunction) or asymptomatic cases, and bulky $(>1 \mathrm{~cm})$, pedunculated highly mobile tumors whether left- or right-sided, though mortality is clearly higher with left-sided CPFs [4-6].

\section{References}

1 Edwards FH, Hale S, Cohen A, et al: Primary cardiac valve tumors. Ann Thorac Surg 1991;52:1127-1131.

2 Neerukonda SK, Jantz RD, Vijay NK, et al: Pulmonary embolization of papillary fibroelastoma arising from the tricuspid valve. Tex Heart Inst J 1991;18:132-135.

3 Mastroberto P, Olivito S, Onorati F, et al: Papillary fibroelastoma of tricuspid valve with pulmonary embolization. Asian Cardiovasc Thorac Ann 2006;14:e53-e54.

4 Ngaage DL, Mullany CJ, Daly RC, et al: Surgical treatment of cardiac papillary fibroelastoma: a single center experience with eighty-eight patients. Ann Thorac Surg 2005;80:1712-1718.

5 Anastacio MM, Moon MR, Damiano Jr RJ, et al: Surgical experience with cardiac papillary fibroelastoma over a 15 year period. Ann Thorac Surg 2012;94:537-541. 
Srivatsa et al.: Multimodality Imaging of Right-Sided (Tricuspid Valve) Papillary Fibroelastoma: Recognition of a Surgically Remediable Disease

6 Gowda RM, Khan IA, Nair CK, et al: Cardiac papillary fibroelastoma: a comprehensive analysis of 725 cases. Am Heart J 2003;146:404-410.

7 Motwani M, Kidambi A, Herzog B, et al: MR Imaging of cardiac tumors and masses: a review of methods and clinical applications. Radiology 2013;268:26-43.
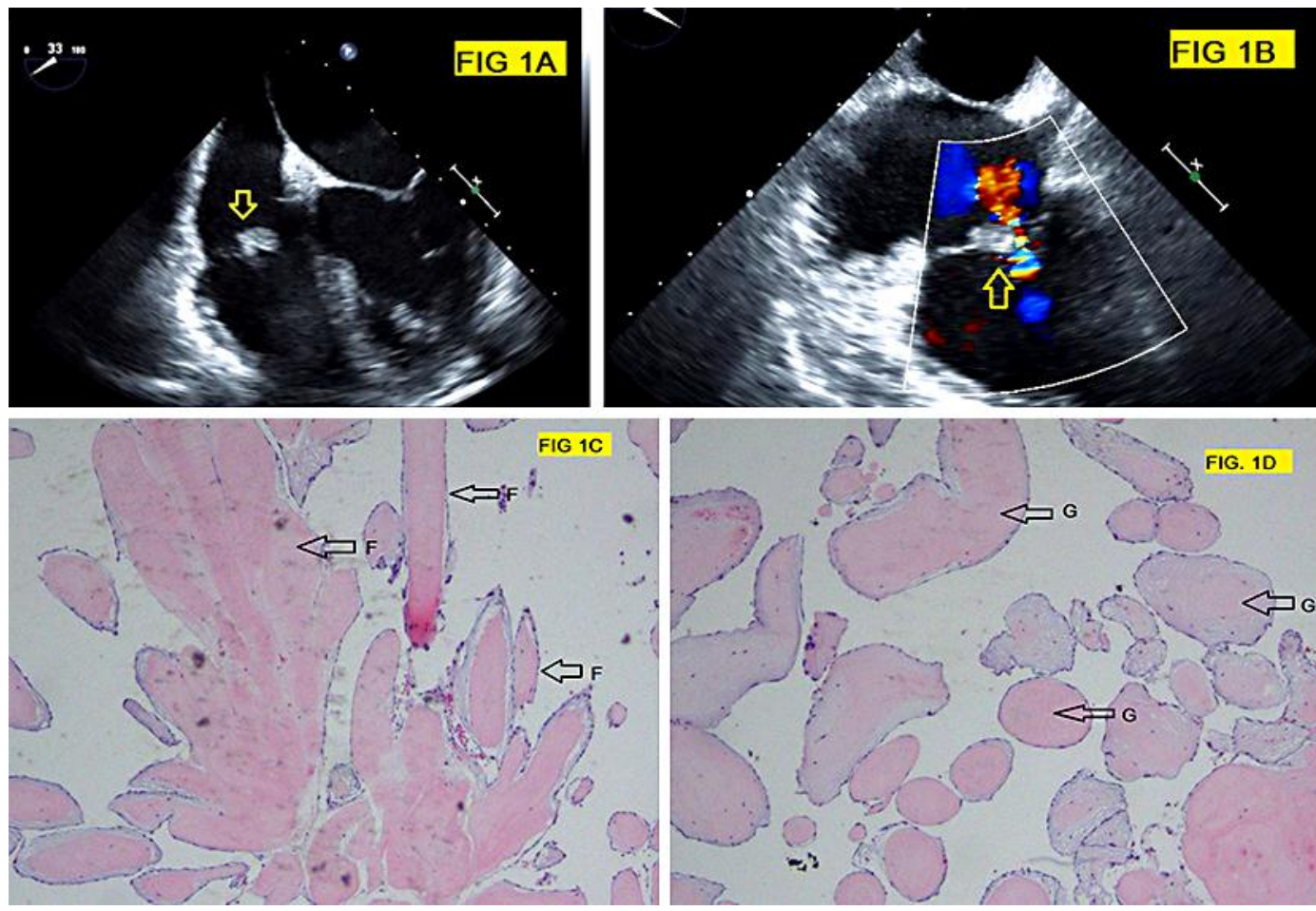

Fig. 1. A, B Apical four-chamber 2D and echocardiographic color flow views. C, D Avascular branching filamentous fronds with a single endothelial cell covering, fibrous core and loose connective tissue rich in mucopolysaccharide.
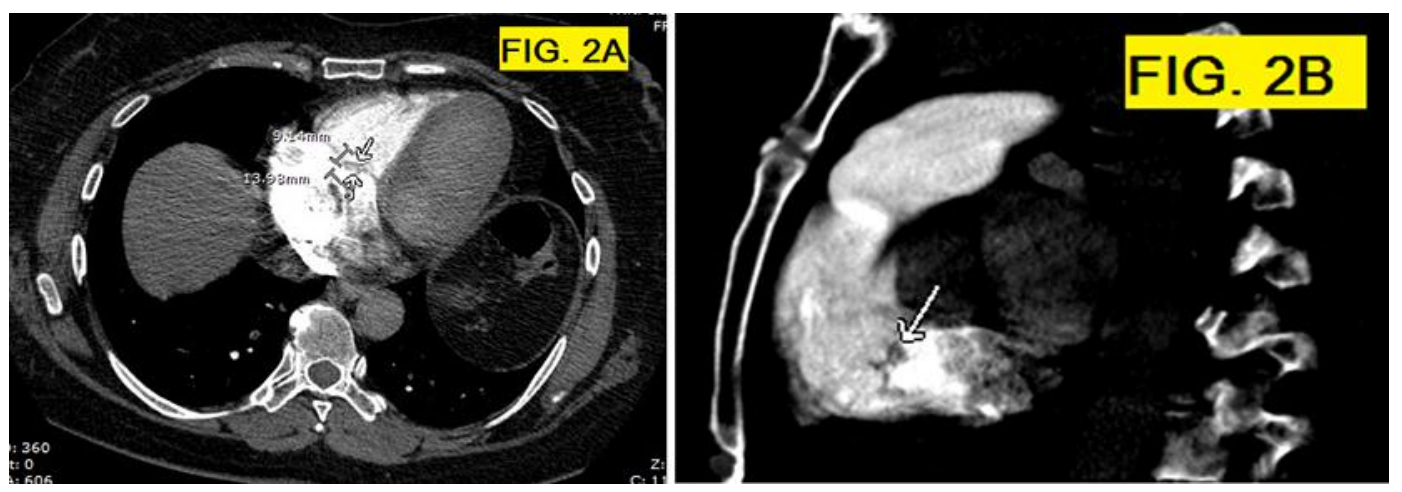

Fig. 2. A, B CT pulmonary angiogram with contrast showing a right-sided TV tumor. 
Srivatsa et al.: Multimodality Imaging of Right-Sided (Tricuspid Valve) Papillary Fibroelastoma: Recognition of a Surgically Remediable Disease

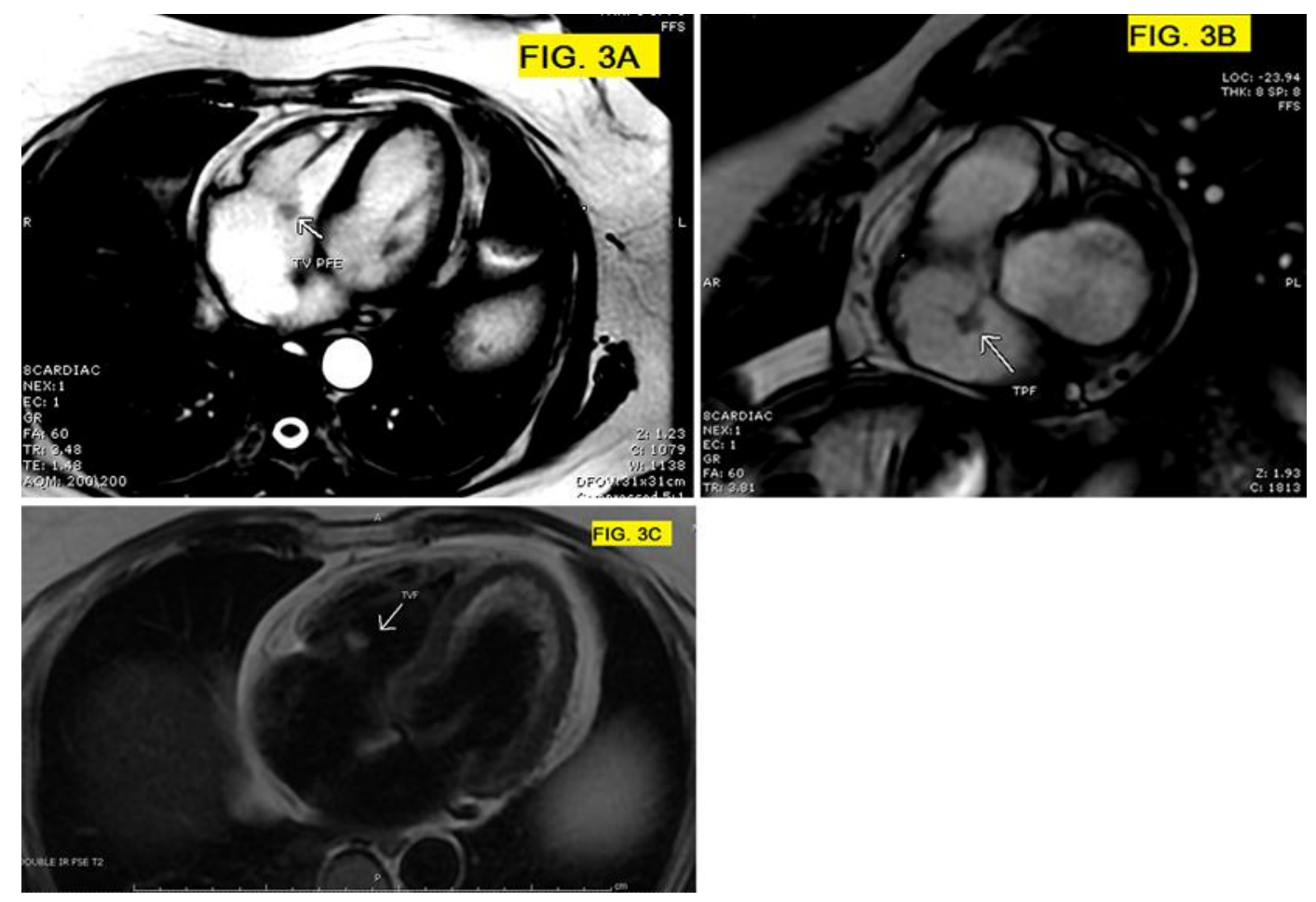

Fig. 3. A TV-PF visualized by FIESTA (bright-blood MRI imaging sequence) with hyperintense signal relative to the myocardium, but hypointensity relative to the blood pool. B Axial FIESTA sequence showing the same pedunculated tumor attached to the TV. C, D Hyperintense tumor signal with inversion recovery $\mathrm{T} 2$ sequences and delayed gadolinium hyperenhancement. 